Beukes et al. Participants' experiences of iCBT for tinnitus

Accepted: 17 August 2018

\title{
Participants' experiences of an Internet-based cognitive behavioural therapy intervention for tinnitus
}

Eldré W. Beukes, ${ }^{1,2}$ Vinaya Manchaiah, ${ }^{2,3,4}$ Alice. S. A. Davies, ${ }^{5,6}$ Peter M. Allen, ${ }^{1,7}$

David M. Baguley, ${ }^{8,9,10}$ \& Gerhard Andersson ${ }^{11,12}$

1. Department of Vision and Hearing Sciences, Anglia Ruskin University, Cambridge, United Kingdom

2. Department of Speech and Hearing Sciences, Lamar University, Beaumont, Texas, USA

3. Department of Speech and Hearing, School of Allied Health Sciences, Manipal University, Karnataka, India

4. Audiology India, Mysore, Karnataka, India

5. College of Human and Health Sciences, Swansea University, Swansea, United Kingdom

6. Audiology Department, Princess of Wales Hospital, Bridgend, United Kingdom

7. Vision and Eye Research Unit, Anglia Ruskin University, Cambridge, United Kingdom

8. National Institute for Health Research, Nottingham Biomedical Research Centre, Ropewalk House, 113 The Ropewalk, Nottingham, United Kingdom

9. Hearing Sciences, Division of Clinical Neuroscience, School of Medicine, University of Nottingham, Nottingham, United Kingdom

10. Nottingham University Hospitals, Nottingham, United Kingdom 
Beukes et al. Participants' experiences of iCBT for tinnitus

11. Department of Behavioural Sciences and Learning, Linköping University, Linköping, Sweden

12. Department of Clinical Neuroscience, Division of Psychiatry, Karolinska Institute, Stockholm, Sweden

\section{Address for correspondence:}

Eldré W. Beukes, Department of Vision and Hearing Sciences, Faculty of Science and Technology, Anglia Ruskin University, Cambridge CB1 1PT, UK. E-mail:

eldre.beukes@anglia.ac.uk; Telephone: +44 (0)1223-698847. 
Beukes et al. Participants' experiences of iCBT for tinnitus

\title{
Participants' experiences of an Internet-based cognitive behavioural therapy intervention for tinnitus
}

\begin{abstract}
Objective This study aimed to explore participants' experiences after undertaking an Internet-based cognitive behavioural therapy intervention (ICBT) for tinnitus.

\section{Design}

Semi-structured telephone interviews were conducted 6-8 months after participants undertook the ICBT intervention. Qualitative thematic analysis was used to interpret the interview data.

\section{Study sample}

A purposeful sampling strategy was used to identify a diverse range of participants. Semistructured interviews were carried out with 15 participants. The mean age was 58.5 years, 7 men and 8 women participated.
\end{abstract}

\section{Results}

The analysis generated the following main themes: (1) expectations and motivation for doing the intervention, (2) experiences of the intervention, (3) intervention engagement and (4) intervention effects. Most participants' expectations were hopeful that the intervention would lessen the impact of their tinnitus. Aspects of the intervention that were beneficial, as well as difficult, were identified together with the impact they had on engagement. Intervention effects were evident on both tinnitus and activities of daily life.

\section{Conclusions}

The benefits described by participants indicate the potential of ICBT as an alternate form of intervention delivery. The difficulties that hampered engagement need to be addressed to enhance the application and to optimise the clinical acceptability of ICBT for tinnitus. 
Beukes et al. Participants' experiences of iCBT for tinnitus

Keywords: semi-structured interviews, Internet-based, cognitive behavioural therapy, tinnitus, qualitative study, experiences

\section{Introduction}

There is a growing emphasis to provide patient-centered healthcare services (Richards, Coulter, and Wicks 2015). Patient-centered care encompasses a holistic healthcare approach together with the provision of personalised, accessible and practical support (Collins 2014). The benefits of patient-centered care include improved quality of care, more appropriate use of healthcare services and reduced onward referrals (Hibbard and Greene 2013; Stacey et al. 2011). It can lead to adoption of healthy behaviours, speed up recovery and improve emotional health (Oates, Weston, and Jordan 2000). Provision of patient-centered care is challenging due to an increased incidence of people living with long-term health conditions (Eaton, Roberts, and Turner 2015). Tinnitus is one such long-term condition, defined as hearing unwanted sounds such as ringing or buzzing in the absence of an external sound source (Baguley, McFerran, and Hall 2013). To meet the demand for tinnitus services, a deeper understanding of the difficulties those with tinnitus are facing is required (Hall et al. 2018). Beukes et al. (2017a) identified the main difficulties those with tinnitus face e.g. difficulty sleeping, listening and concentrating. To further this work, Manchaiah et al. (2018), studied the impact of tinnitus on daily functioning using the International Classification of Functioning, Disability and Health (ICF) framework. Similar results were identified, indicating that tinnitus impacted on sleep, hearing, attention and mood. Experiencing tinnitus also led to activity limitations and participation restrictions when communicating and socialising. Tinnitus interventions should target improving these difficulties. The provision of coping strategies and support networks can result in personal development and a positive 
Beukes et al. Participants' experiences of iCBT for tinnitus

outlook despite experiencing tinnitus (Beukes et al. 2017c). This provision and support is, however, not always readily available (McFerran et al. 2018). To improve access to tinnitus interventions, an Internet-based cognitive behavioural therapy (ICBT) was developed in Sweden (Andersson et al. 2002) and subsequently been adapted for different cultures (Jasper et al. 2014; Beukes et al. 2016). The efficacy of ICT for tinnitus has been indicated (Anderson, 2015) and overall satisfaction with the intervention has been high (Beukes et al. 2016; Beukes et al. 2017b). Although clinical outcomes and user satisfaction indicate potential of ICBT, participants' experiences of this intervention have not been evaluated. There have been relatively few studies investigating participant experiences with ICBT for other conditions such as depression (e.g. Ly et al. 2015; Beattie et al. 2009; Bendelin et al. 2011). Only two studies have focused on experiences of audiological Internet-based interventions. Participants who undertook an Internet-based aural rehabilitation (IAR) intervention found it of value due to an increase in knowledge (Malmberg et al. 2018). . Those who underwent an Internet-based vestibular rehabilitation intervention reported that it was easy to use and promoted engagement with the rehabilitation exercises (Essery et al. 2017). In order to fill the knowledge gap relating to experiences with ICBT for tinnitus the present study was undertaken. A detailed understanding of participants' perceptions of ICBT is important to determine its potential as a patient-centered approach.

\section{Materials and methods}

\section{Study design}

ICBT is classified as a complex intervention because it involves a number of interacting components (Craig et al. 2008). To explore some of these complexities and to increase understanding of the way ICBT for tinnitus is experienced, a qualitative study design was selected. Qualitative studies can provide a rich data set by an in-depth exploration of 
Beukes et al. Participants' experiences of iCBT for tinnitus

individual experiences (Fossey et al. 2002). Semi-structured telephone interviews were used to obtain qualitative information regarding participants' experiences of ICBT for tinnitus.

The COREQ checklist (Tong, Sainsbury, and Craig 2007) was followed for the design and analysis of this study (see Supplementary Appendix 1). Ethical approval was granted by Anglia Ruskin University in Cambridge, UK (FST/ FREP/14/478).

\section{Sampling and recruitment}

The participants from which the sample was drawn consisted of those who had previously undertaken a guided ICBT intervention for tinnitus (Beukes et al. 2018). Recruitment for this study was via advertisement, largely through social media. In the original study 146 participants were randomised into either an experimental group, receiving the ICBT treatment, or the control weekly check-in group, who completed a weekly questionnaire during the active treatment phase before undertaking the iCBT treatment. Results from the RCT indicated the efficacy of iCBT in reducing tinnitus distress and some of the tinnitusrelated difficulties up to one year post-intervention (Beukes et al. in press).

For the present study a diverse sample of participants from the original RCT were sought. A purposeful sampling strategy, namely maximum variation, was used to obtain a heterogeneous sample (Teddlie and Yu 2007). As participants from the experimental group and the control group may have had varying experiences, the aim was to select participants from both groups. Participants with varying demographical profiles were also sought. Participants who undertook the original clinical trial were thus stratified according to gender (male or female); group randomised as per the RCT (experimental or control); preintervention tinnitus severity score as measured by the Tinnitus Functional Index (Meikle et al. 2012) to be either low (below 50) or high (above 50 out of 100); and the number of years they have had tinnitus (less than or more than 10 years). A participant from each category 
Beukes et al. Participants' experiences of iCBT for tinnitus

was invited at random via email to participate in a telephone interview. Two reminder emails were sent and if a participant did not respond an alternative participant from the same category was randomly contacted.

\section{Data collection}

Semi-structured interviews were used to explore the experiences of participants. Open-ended questions were asked in a single telephone interview. One interviewer interviewed all the participants. The interviews were recorded for analysis purposes and notes were taken during the interview. The average duration of the telephone call was 13 minutes (range 9-16 minutes). Participants were telephoned 6-8 months following completion of the treatment, to ensure the main themes identified would be the overall impressions remaining after some distance from the intervention.

A broad interview guide was prepared comprising of 10 questions as a tool to elicit information (see Supplementary Appendix 2). Interviews were transcribed and participants were given copies via email and asked to make corrections as required. Participants were not involved in providing further feedback regarding the findings or results.

\section{Interviewer}

The interviewer (AD) was a female Clinical Audiologist at a National Healthcare Hospital and a university lecturer with experience of assessing and treating patients with tinnitus. Although personal characteristics may introduce bias, this was minimised by selecting an interviewer who was independent from previous research related to ICBT for tinnitus. The interviewer was also unaware of the participants' outcomes, study group and any demographical information to further minimise personal bias. To identify personal bias the process of reflexivity was applied which involves identifying the interviewer's possible influence during the interview. The interviewer was interested in finding out more about 
Beukes et al. Participants' experiences of iCBT for tinnitus

alternative formats of providing counselling-based information for those with hearing-related difficulties. She, however, did not have any strong views on this form of intervention. Her experience working face-to-face with patients, made her potentially biased toward a face-toface intervention approach. She was, however, open to evaluating whether other approaches may work for patients as she has previously investigated whether instructional DVDs were beneficial in reducing reported hearing aid problems.

\section{Pilot study}

Prior to the main study, semi-structured interviews were conducted with two participants as a pilot study. This was to ensure that the interviewer was familiar with the protocol and that the recordings were clear enough to transcribe. During the pilot study one recording was too poor to transcribe and the other recording file became corrupt. Adjustments were made to the procedures to overcome these problems and ensure the smooth-running of the main interviews.

\section{Data analysis}

Qualitative data coding was performed using QSR International's NVivo 11 Software (QSR International Pty Ltd 2010). Qualitative thematic analysis forms the theoretical framework to systematically identify, analyze and report patterns within data into a structured format (Braun and Clarke 2006). Thematic analysis was selected because of its ability to detect patterns of meaning that would facilitate understanding of the data (Braun and Clarke 2006). This analysis examined the data in a recursive process to identify a set of condensed categories. These categories were derived inductively using a bottom-up approach without imposing advanced pre-conceived categories or theoretical perspectives (deductively). Initially the verbal data were transcribed into the written form. Transcriptions were actively 
Beukes et al. Participants' experiences of iCBT for tinnitus

read and re-read in search of initial ideas, meanings and patterns. These responses were coded for "meaning units", which are the statements that relate to the same central category and formed the units of analysis for coding. Meaning units were then sorted into potential candidate themes and later sub-themes. Categories were gradually condensed by combining those with similarities to ensure they were mutually exclusive. Category labels were assigned by selecting meaningful terms. After selecting the codes and categories the original data were rechecked for consistency and to ensure responses were appropriately categorised.

Initial data coding was performed by EB and cross-checked by two additional authors (VM and $\mathrm{AD}$ ) to reduce possible research bias and improve reliability. The final categories were selected where there was agreement between two out of the three researchers.

\section{Results}

\section{Participants}

Of the 23 targeted participants, 2 declined and 6 did not respond to the recruitment e-mail. After 13 participants had been interviewed data saturation was reached as no new insights emerged. Two further participants were interviewed and confirmed data saturation, thus no further participants were interviewed.

There were 7 men and 8 women who participated with a mean age of 58.5 years (SD: 8.2) and mean tinnitus duration of 9.4 years (SD: 9.0). There were 7 participants from the original experimental group (undertook the iCBT intervention initially) and 8 participants were from the original control group (had an 8 week delay before undertaking the intervention). Purposeful sampling was effective at identifying a heterogeneous sample as indicated in Table 1.

\section{Table 1: Participant characteristics}


Beukes et al. Participants' experiences of iCBT for tinnitus

\begin{tabular}{|c|c|c|c|c|c|c|c|}
\hline \multirow{3}{*}{ Number } & \multirow{3}{*}{ Group } & \multirow{3}{*}{ Gender } & \multirow{3}{*}{ Age } & \multirow{3}{*}{$\begin{array}{l}\text { Tinnitus } \\
\text { Duration }\end{array}$} & \multirow{3}{*}{$\begin{array}{l}\text { TFI } \\
\text { pre }\end{array}$} & \multirow{3}{*}{$\begin{array}{l}\text { TFI } \\
\text { post }\end{array}$} & \multirow{3}{*}{$\begin{array}{l}\text { No. } \\
\text { of } \\
\text { logins }\end{array}$} \\
\hline & & & & & & & \\
\hline & & & & & & & \\
\hline 1 & Experimental & female & 64 & 10 & 37 & 20 & 38 \\
\hline 2 & Experimental & male & 45 & 3 & 52 & 13 & 50 \\
\hline 3 & Experimental & female & 47 & 0.7 & 54 & 26 & 27 \\
\hline 4 & Experimental & male & 57 & 4.5 & 42 & 23 & 27 \\
\hline 5 & Experimental & male & 57 & 15 & 37 & 6 & 37 \\
\hline 6 & Experimental & female & 52 & 0.4 & 84 & 48 & 52 \\
\hline 7 & Experimental & female & 58 & 2 & 50 & 14 & 26 \\
\hline 8 & Control & male & 73 & 3 & 68 & 36 & 34 \\
\hline 9 & Control & female & 54 & 6 & 75 & 65 & 19 \\
\hline 10 & Control & female & 71 & 35 & 38 & 19 & 30 \\
\hline 11 & Control & male & 61 & 15 & 63 & 42 & 39 \\
\hline 12 & Control & female & 66 & 11 & 74 & 12 & 73 \\
\hline 13 & Control & female & 56 & 7 & 74 & 10 & 43 \\
\hline 14 & Control & male & 52 & 14 & 56 & 35 & 52 \\
\hline 15 & Control & male & 65 & 15 & 70 & 32 & 14 \\
\hline
\end{tabular}

Acronyms. TFI: Tinnitus Functional Index Score. Logins: Number of logins during the 8week intervention

\section{Identified Themes}

The words most frequently used during the interviews were related to "thinking/thoughts" and "relaxation" (Figure 1). This was related to participants mentioning a positive change in thinking patterns and the ability to relax post-intervention. The words "helped, positive, 
Beukes et al. Participants' experiences of iCBT for tinnitus

different" were also often mentioned in terms of experiences with the intervention. The most frequently used words and deeper analysis of the responses led to the identification of four overarching themes namely (1) expectations and motivation, (2) experiences, (3) engagement and (4) intervention effects (Figure 2). These will each be discussed in turn.

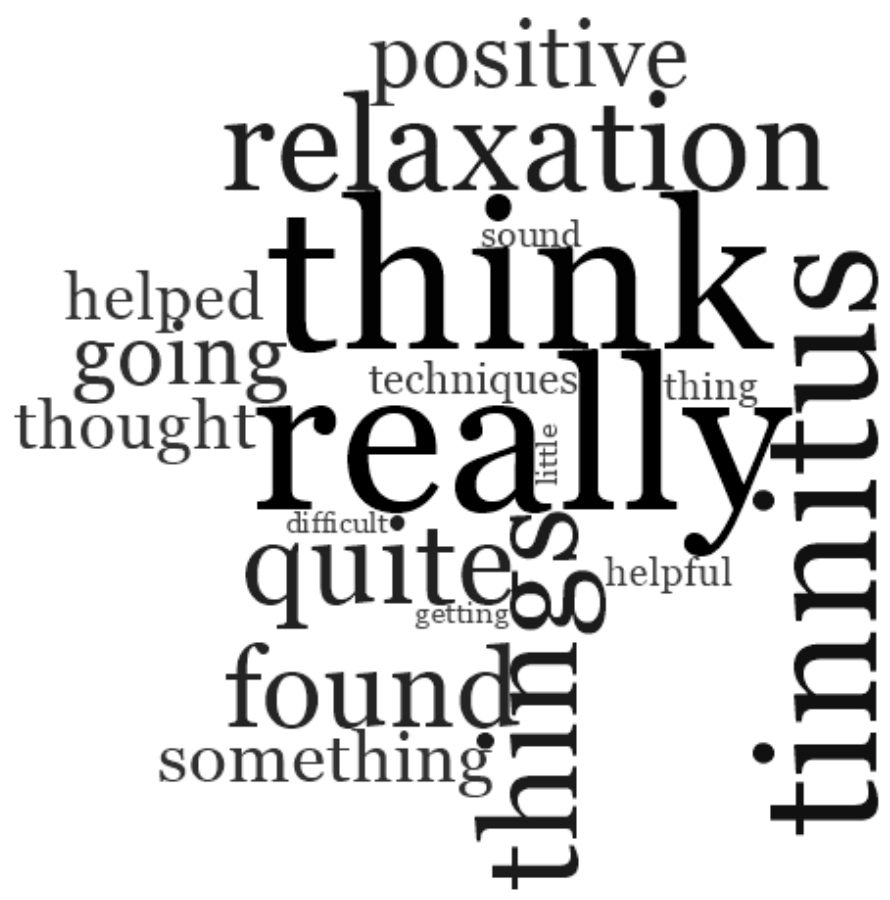

Figure 1. Word cloud of the most frequently used words when describing the experiences with the intervention. A word that stands out more was more highly represented. 


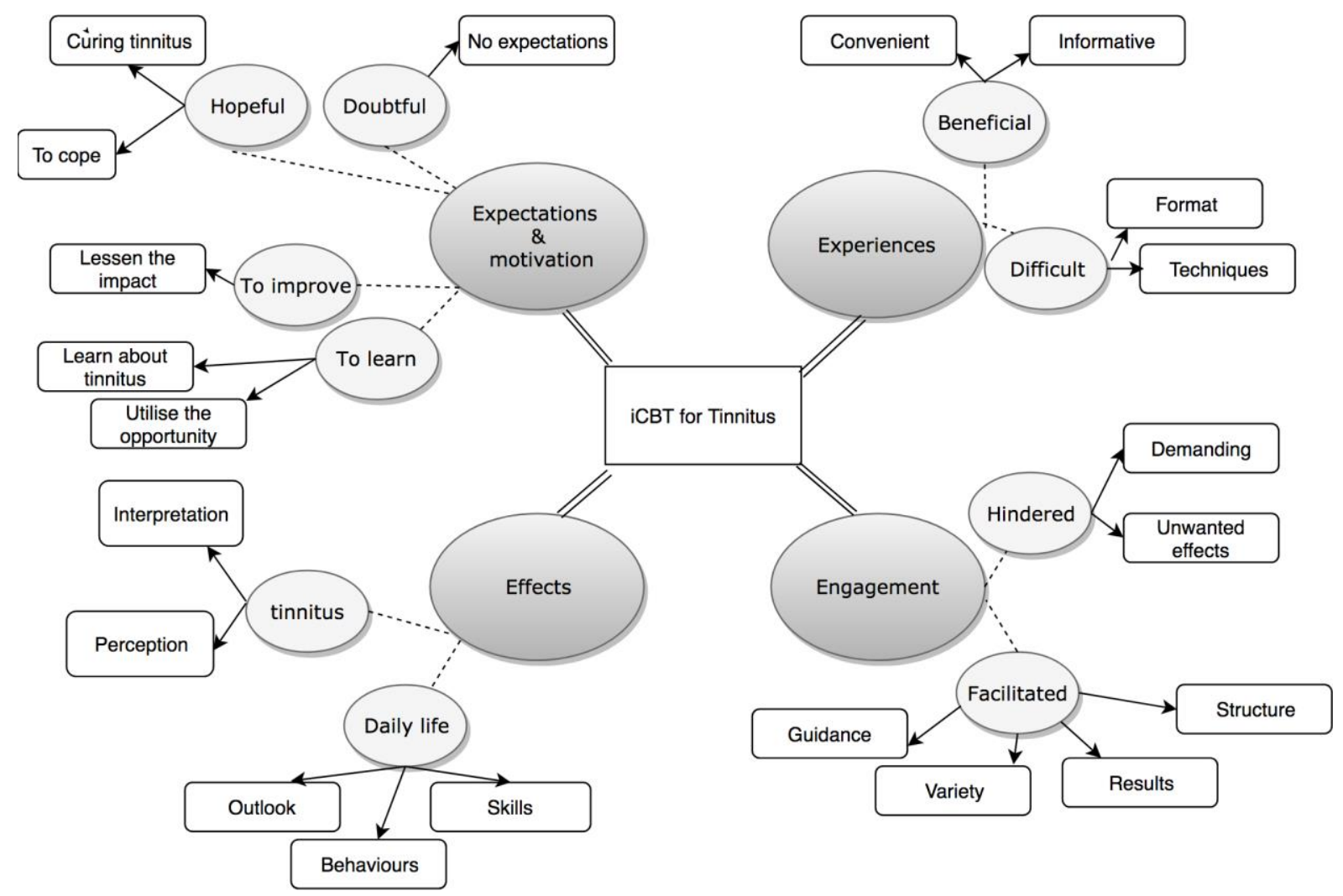

Figure 2. Summary of the identified themes and sub-themes regarding participant experiences with iCBT for tinnitus

\section{Expectations and motivation to undertake the intervention}

The first overarching theme that was identified was participants' expectations and their motivation for doing the intervention (Table 2). Many participants were hopeful that the intervention may help them better cope with their tinnitus. Others were curious about how the intervention would work. Some were doubtful that this intervention format would provide any benefit. .

Motivation to undertake the intervention included wanting to lessen the impact and utilise the opportunity to learn more about tinnitus.

\section{Table 2: Pre-intervention expectations and motivation}


Beukes et al. Participants' experiences of iCBT for tinnitus

\begin{tabular}{|c|c|c|c|}
\hline Theme & Sub-theme & $\begin{array}{l}\text { Number } \\
\text { of } \\
\text { meaning } \\
\text { units }\end{array}$ & Example of a meaning unit \\
\hline Pre-inter & ention expec & ations & \\
\hline Hopeful & Curiosity & 8 & $\begin{array}{l}\text { "I was hoping that it would help me in ways to } \\
\text { deal with the tinnitus as I have suffered with it } \\
\text { quite a lot at the time." } \\
\text { "Well, I was sort of intrigued I think to see how it } \\
\text { would work. I didn't really have any definite } \\
\text { ideas." }\end{array}$ \\
\hline Doubtful & None & 3 & $\begin{array}{l}\text { "I wasn't hopeful. Probably, if I had been going } \\
\text { to see a clinician, I would have felt more hopeful. } \\
\text { I expected nothing because I had tried everything } \\
\text { before and nothing worked." }\end{array}$ \\
\hline
\end{tabular}

\section{Motivation to do the intervention}

\begin{tabular}{|c|c|c|c|}
\hline $\begin{array}{l}\text { To } \\
\text { improve }\end{array}$ & $\begin{array}{l}\text { Lessen the } \\
\text { impact }\end{array}$ & 6 & $\begin{array}{l}\text { "I was motivated because the noise was driving } \\
\text { me insane. It would ruin my enjoyment of most } \\
\text { days. I feel quite depressed about it." }\end{array}$ \\
\hline To learn & $\begin{array}{l}\text { Learn } \\
\text { about } \\
\text { tinnitus }\end{array}$ & 2 & $\begin{array}{l}\text { "What motivated me was the fact that I wanted to } \\
\text { find out more about tinnitus. I had already read a } \\
\text { lot about it before, but I wanted to know what this } \\
\text { particular programmes take was." }\end{array}$ \\
\hline
\end{tabular}


Beukes et al. Participants' experiences of iCBT for tinnitus

Utilise the
opportunity
the opportunity. I had been given an opportunity
and I didn't want to waste it."

\section{Experiences of undertaking the intervention}

A further overarching theme was participants' experiences of the intervention (Table 3).

They indicated that the treatment was beneficial and enjoyable to do. They found it convenient in terms of ease of accessibility, privacy offered and flexibility to do at any time.

Some described that being able to do the intervention at home minimised the stress associated with attending hospital appointments. Participants indicated that difficult aspects of the intervention were related to the way the worksheets were structured and the pace of the intervention. They also found some techniques difficult to do such as deep relaxation, visualisation, exposure to tinnitus and identifying unhelpful thoughts.

Table 3: Experiences of undertaking the intervention

\begin{tabular}{llll}
\hline Theme & Sub-theme & Number & Example of a meaning unit \\
& & \\
& & \\
& & \\
& meaning & \\
\hline Beneficial & & & \\
\hline Convenient & Accessible & 11 & "It was accessible. You don't have to \\
& & & travel miles and wait for an appointment, \\
& & & it's there for you."
\end{tabular}


Beukes et al. Participants' experiences of iCBT for tinnitus

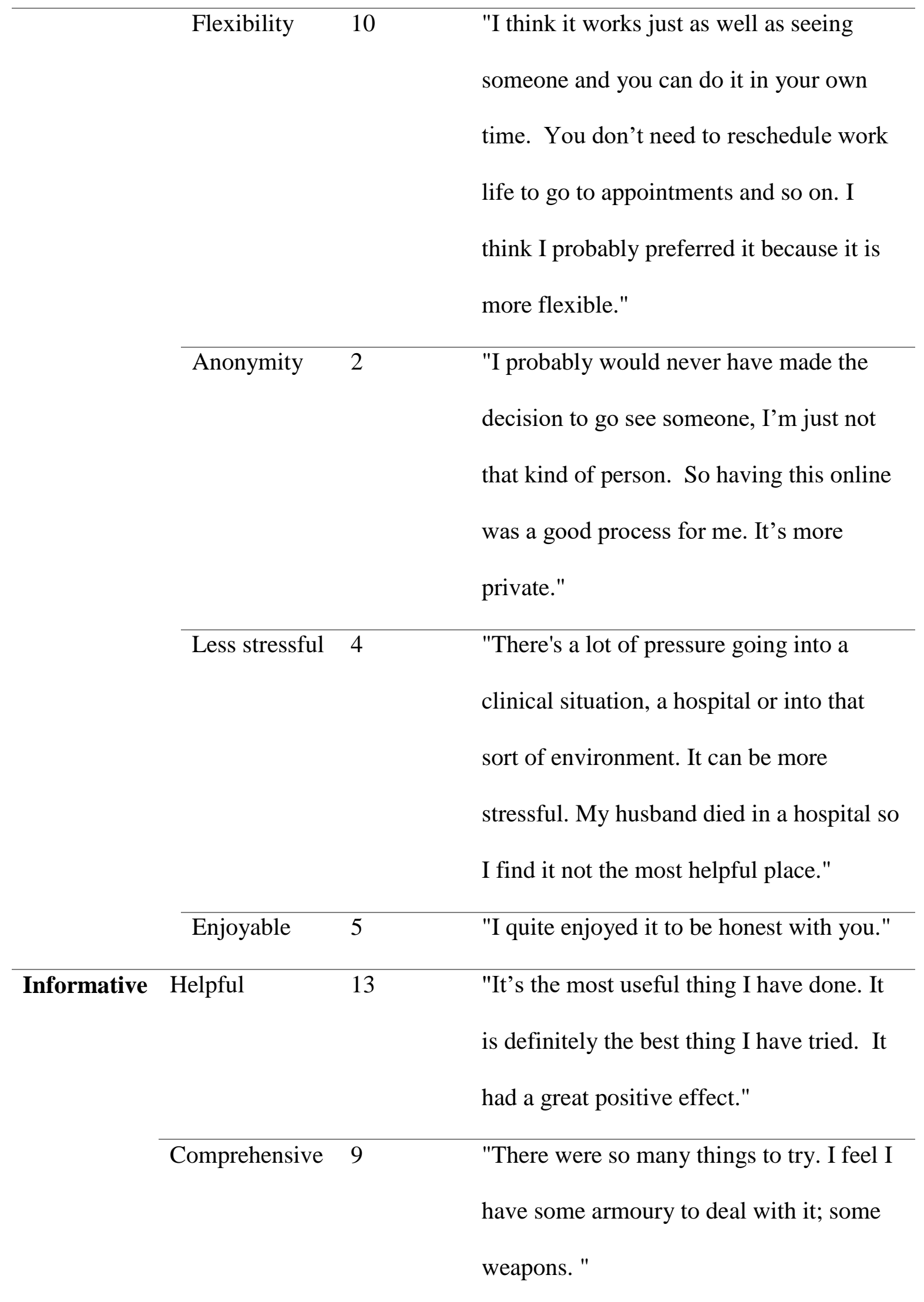

\section{Difficult}


Beukes et al. Participants' experiences of iCBT for tinnitus

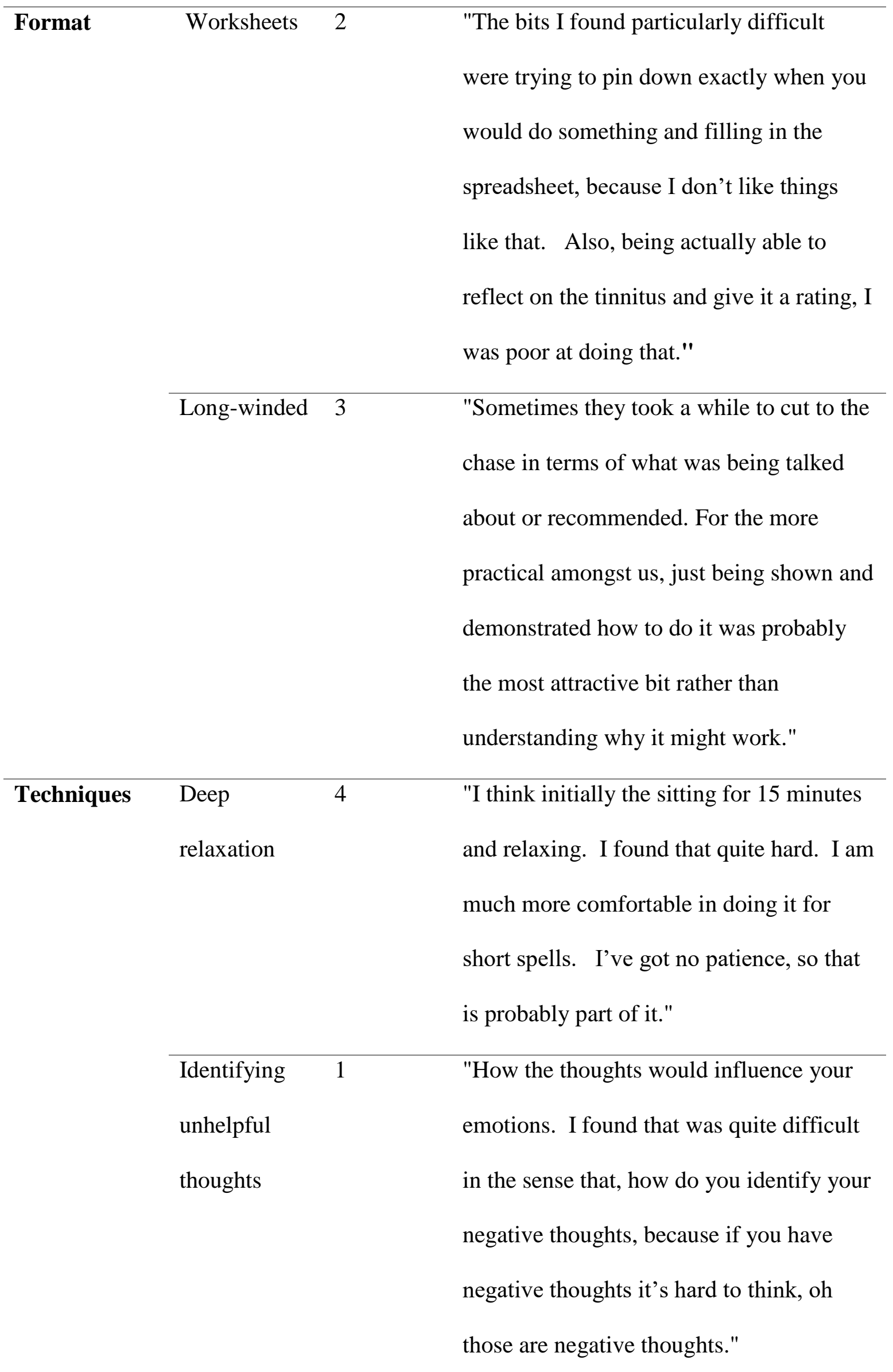


Beukes et al. Participants' experiences of iCBT for tinnitus

$\begin{array}{lll}\begin{array}{l}\text { Exposure to } \\ \text { tinnitus }\end{array} & \text { "There was an exercise where you had to } \\ & \text { tune in to listen to your tinnitus and I } \\ \text { found that really difficult." }\end{array}$

\section{Factors affecting engagement}

Factors that facilitated and hindered engagement was a further overarching theme (Table 4). Engagement was facilitated by the guidance provided, the variety of different modules and noticing improvement in symptoms. The structure of the intervention promoted engagement. This was due to the ability to work autonomously, review previous modules, having reminder tips and the regular weekly input. Engagement was hindered by the time required to do the intervention and that short summary notes regarding the techniques were not available. Engagement was also hampered by the initially worsening of tinnitus due to the focusing on it.

Table 4. Factors affecting engagement

\begin{tabular}{|c|c|c|c|}
\hline Theme & Sub-theme & & $\begin{array}{l}\text { Number Example of a meaning unit } \\
\text { of } \\
\text { meaning } \\
\text { units }\end{array}$ \\
\hline Facilitated & & & \\
\hline Guidance & Messages & 3 & $\begin{array}{l}\text { "But the other part was down to the emails I received, } \\
\text { usually once or twice a week. They were so }\end{array}$ \\
\hline
\end{tabular}


Beukes et al. Participants' experiences of iCBT for tinnitus

encouraging that they would have kept me going, even if I didn’t want to."

\begin{tabular}{lll}
\hline Results & Progress & "...then it began to work. There's nothing more \\
& motivational that something starting to work. I don't \\
& think I ever had a problem with engaging with the \\
& programme overall." \\
\hline Variety $\quad 5$ & "Knowing there was another chapter coming along, I \\
& carried on feeling intrigued, if you like, to know what \\
& was coming next. It was all new to me. Different \\
& ways of coping, which I never knew about, and they \\
& helped"
\end{tabular}

Structure Self-help 2 "It was sort of like self-help. It's up to me, you have more control over the experience. For me, I'm a bit of an introvert so actually being able to do things quietly on my own works for me. "

Regularity 4 "Overall, I liked the structure and style of communication. I liked the regularity of the weekly check-ins and questionnaires. having that pattern set every week helped me maintain working through it" Reflecting 3 "Overall, I think that the way the modules were structured in terms of developing techniques and understanding kind of in tandem was a good way of doing it. Once you are able to look back, you can see how they build on each other. I liked going back and 
Beukes et al. Participants' experiences of iCBT for tinnitus

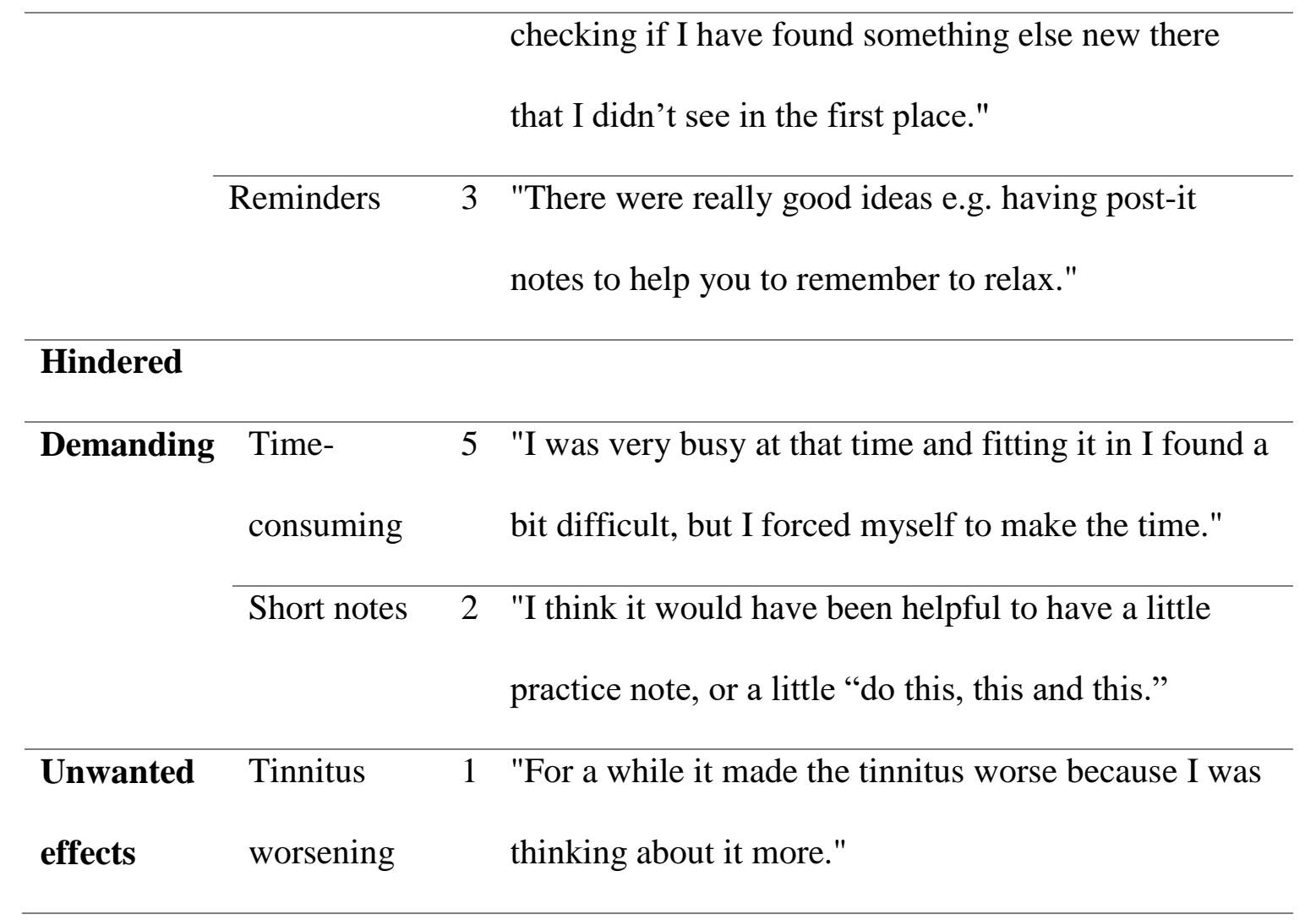

\section{Post-intervention effects}

A further theme that was identified was the impact of the intervention on both tinnitus and daily life (Table 5). Participants were more acceptant of their tinnitus and it became less noticeable over time. They had reinterpreted the tinnitus and had a more positive perception thereof. The associated fears had decreased and they realised that they are not the only ones with tinnitus. Moreover, the intervention had affected daily life in terms of their outlook being more positive and having fewer avoidance behaviours. Their sleep quality and ability to relax also improved. They also indicated having developed new skills to aid problemsolving and manage stress and anxiety.

\section{Table 5 Post-intervention effects}


Beukes et al. Participants' experiences of iCBT for tinnitus

\begin{tabular}{|c|c|c|c|}
\hline Theme & Sub-theme & $\begin{array}{r}\text { Number } \\
\text { of } \\
\text { meaning } \\
\text { units }\end{array}$ & Example of a meaning unit \\
\hline Interpretation & Accepting & 7 & $\begin{array}{l}\text { "One particular bit I did get out of it which } \\
\text { helped me a great deal, was acceptance. } \\
\text { I'd never thought of it before, but just } \\
\text { simply accepting that my body has } \\
\text { decided to make this hiss and I've got to } \\
\text { live with it. It's no good fighting it or } \\
\text { ranting against it. I just accepted it. I } \\
\text { must say that, that was the little jewel that } \\
\text { I have got out of this tinnitus programme." }\end{array}$ \\
\hline & Reactions & 11 & $\begin{array}{l}\text { "Now my emotional reaction is different. } \\
\text { It took away a lot of the fear and stress } \\
\text { associated with the tinnitus. I'm not as } \\
\text { afraid of it as I used to be. I don't feel it's } \\
\text { so scary anymore, I'm not frightened of } \\
\text { it." }\end{array}$ \\
\hline Perception & Reinterpreted & 6 & $\begin{array}{l}\text { "For me the really critical module was the } \\
\text { reinterpretation module. When it is bad I } \\
\text { imagine going for a walk, raindrops } \\
\text { pouring down a window pane, things like } \\
\text { that; and that helped change the way I see } \\
\text { my tinnitus." }\end{array}$ \\
\hline
\end{tabular}


Beukes et al. Participants' experiences of iCBT for tinnitus

\begin{tabular}{lll}
\hline Less & 2 & "I would say that the majority of the time \\
noticeable & now I'm not conscious of it, although it is \\
& still there." \\
\hline Controlled & 7 & "I can cope now. Even if it is bad I can do \\
& something about it. It's not controlling me \\
& like it used to." \\
\hline Less alone & "You realise that you are not alone, there \\
& are thousands of people who suffer from \\
& this."
\end{tabular}

\section{Daily Life}

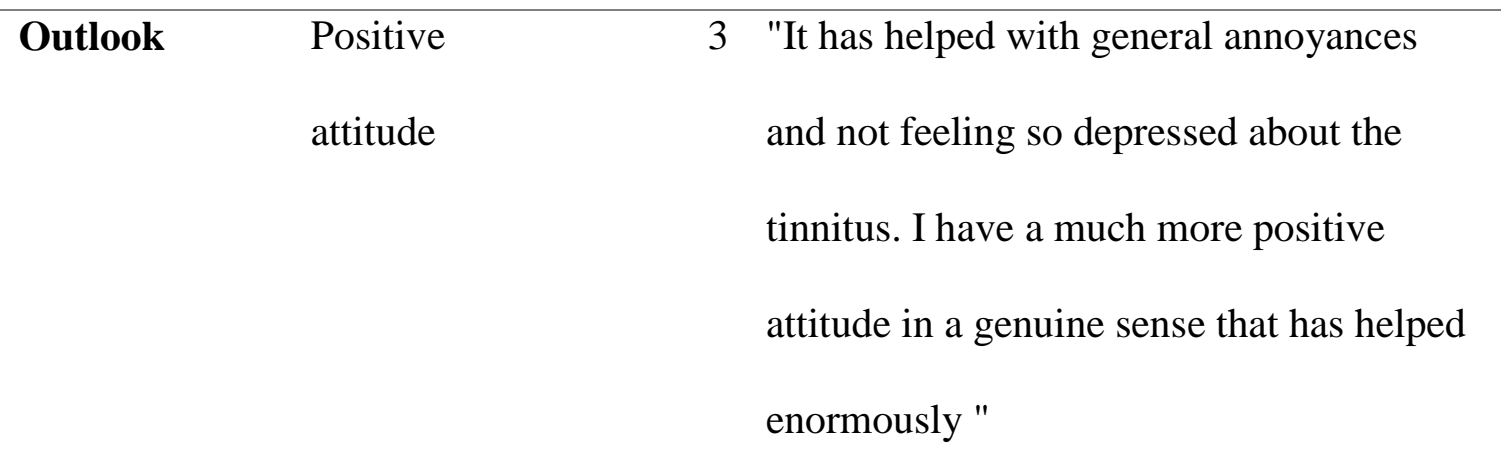

\begin{tabular}{lll}
\hline Behaviours Sleep & "It has been absolutely brilliant, especially \\
& as far as sleeping. I think if one sleeps \\
& properly or at least improves ones sleep \\
& pattern that is a huge help on tackling the \\
& rest of your life." \\
& "Now my tinnitus doesn't stop me doing \\
& avoidance & anything, which it was doing. I mean, I \\
& stopped visiting friends and going out. I \\
& used to go and listen to bands and I just
\end{tabular}


Beukes et al. Participants' experiences of iCBT for tinnitus

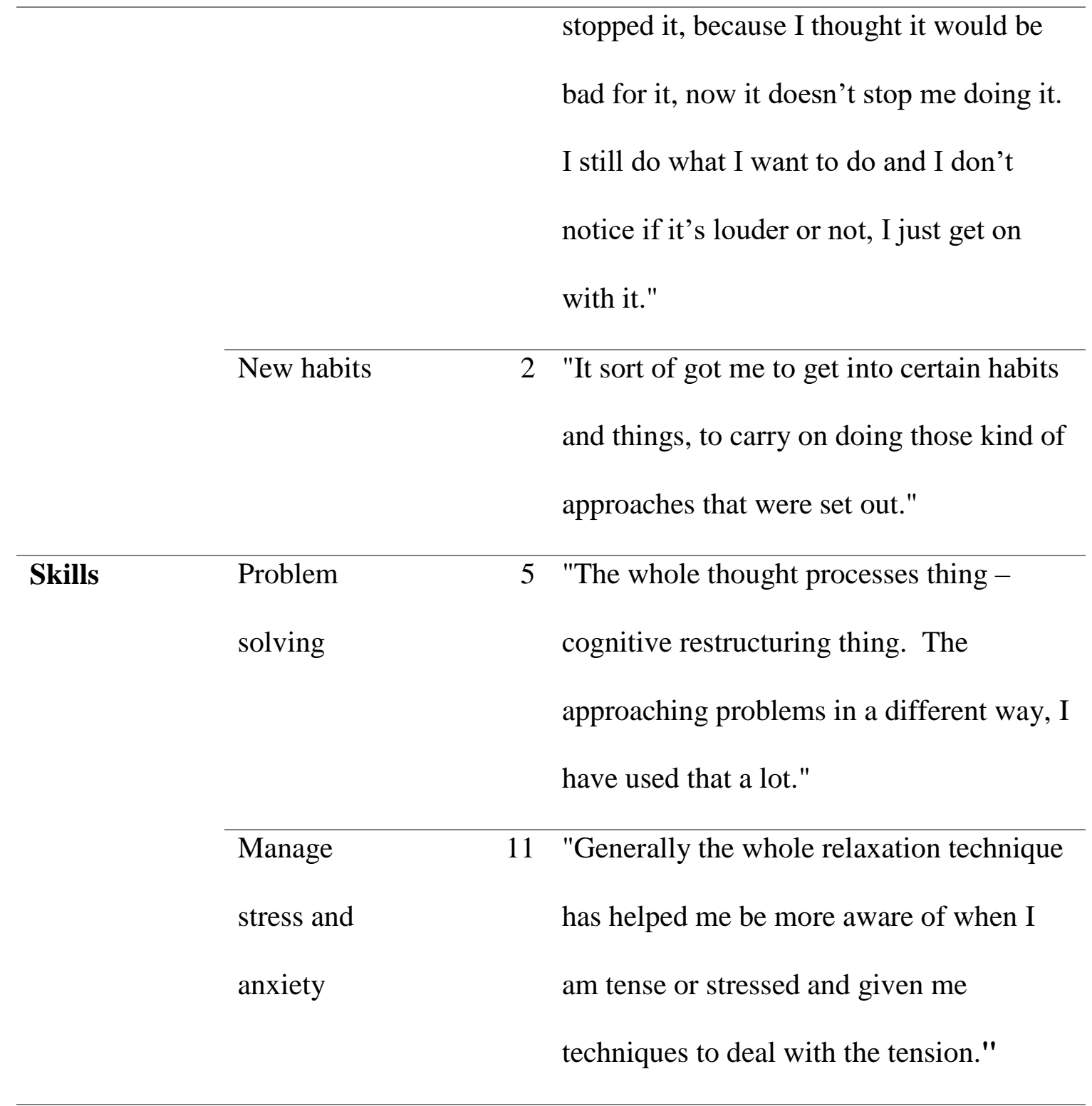

\section{Discussion}

The aim of this qualitative study was to capture participants' experiences after undertaking ICBT for tinnitus. Semi-structured telephone interviews were conducted with 15 participants. Qualitative thematic analysis was used to analyse the transcripts. This discussion explores the main themes identified. 
Beukes et al. Participants' experiences of iCBT for tinnitus

\section{Experiences related to expectations and motivation to do the intervention}

In many of the interviews, participants described the devastating effects tinnitus had had on their lives. Some were hopeful that undertaking the intervention would help them reduce the impact tinnitus had. Others were intrigued by what the intervention had to offer. The possibility of learning new techniques and more about tinnitus motivated participants to undertake the intervention. Some were initially doubtful that undertaking the intervention would help. This was related to having tried other interventions and not finding these beneficial. Expectations of an IAR intervention were also generally of curiosity and hope of improvement (Malmberg et al. 2018). Wider research has identified that treatment credibility and motivation can positively affect intervention engagement and outcomes (Bendelin et al. 2011). Ways of improving treatment credibility of ICBT for tinnitus should thus be considered. If patients view Internet-interventions as credible, this may aid motivation to consider undertaking Internet-interventions and positively influence intervention engagement.

\section{Experiences undertaking the intervention}

Participants mentioned a range of both beneficial and difficult experiences while undertaking the intervention. Diverse experiences have also been reported regarding ICBT for depression (Holst et al. 2017). In the present study, participants found the intervention beneficial in terms of ease of access, privacy, weekly input and the flexibility to do it at home. Some of these factors have been identified before (Andersson and Titov 2014; Beattie et al. 2009). Holst et al. (2017) and Malmberg et al. (2018) also reported that participants valued being able to revisit materials, the flexibility the intervention offers and having ease of access to information. One unique finding from the present study that has not been emphasised in prior research is that some that some individuals find attending clinics or hospitals stressful. Minimising stress and anxiety are important in the management of tinnitus. Providing 
Beukes et al. Participants' experiences of iCBT for tinnitus

patients with alternative options should be prioritised as a means of patient-centred care. Participants explained that the intervention content was easy to follow, helpful, informative and comprehensive. Some found that there was too much information and would have preferred a shorter intervention format. Finding a balance between a comprehensive intervention and information overload is difficult to achieve. Simplifying the intervention worksheets may be one way of addressing some of the difficulties mentioned. The weekly assignment and time commitments were also identified as a negative experience by some undertaking an IAR intervention (Malmberg et al. 2018) and Internet-based vestibular rehabilitation (Essery et al. 2018). Divergent experiences were evident as some participants mentioned finding certain techniques useful whereas others found the same techniques difficult to do. Due to the heterogeneous nature of tinnitus, having a range of techniques for participants is important. Highlighting the rationale for each technique and that not all techniques may be effective for each individual is important.

\section{Experiences affecting intervention engagement}

Participants mentioned factors that both promoted and hindered intervention engagement. Engagement was promoted by the ability to take control of their own treatment, the regularity of the modules and being able to review the module content. Participants who undertook an Internet-based vestibular rehabilitation intervention reported that similar factors facilitated engagement, such as perceived symptom reduction, ease of use of the intervention, and becoming self-sufficient (Essery et al. 2017). Identifying small improvements promoted engagement, as also found by Bendelin et al. (2011) in ICBT for depression and Heinrich et al. (2016) in ICBT for tinnitus. In the present study, the guidance provided was mentioned most frequently as a factor that encouraged engagement. The motivation, encouragement and reassurance provided by guidance during self-help interventions has also previously been 
Beukes et al. Participants' experiences of iCBT for tinnitus

reported to enhance engagement (e.g. Muller, Kirby, and Yardley 2015). Guided interventions lead to better outcomes than un-guided interventions (Baumeister et al. 2014).

Engagement was hampered by a lack of time to do the intervention. Johansson et al. (2015) also identified that certain ICBT treatment features such as the workload or textcontext complexity could contribute to non-engagement. Unwanted intervention effects such as an initial worsening of tinnitus further impeded engagement. Ways of lessening the intervention demands and possible unwanted effects related to such an intervention should be sought to facilitate effective engagement and promote effective outcomes (O'Connor et al. 2016; Yardley et al. 2016).

\section{Intervention effects}

Undertaking the intervention had a positive impact on tinnitus perceptions and daily life. The theoretical underpinnings of the intervention were based on cognitive behavioural therapy techniques to target reactions, behaviours and thoughts surrounding tinnitus (Andersson 2002). The intervention was successful at helping participants reinterpret their tinnitus and thus have a more positive reaction toward the tinnitus. Participants also became more acceptant of the tinnitus and found that it became less noticeable. Although analysis of tinnitus-distress using self-reported assessment measures post-intervention indicated an improvement, the reasons for this improvements were not identified during quantitative analysis. Results from the present study indicate that although the tinnitus remained, the intervention aided acceptance and reinterpretation of the tinnitus, which strengthens the efficacy of ICBT in reducing tinnitus distress (Andersson et al. 2015).

The intervention effects were also evident in daily life. Bendelin et al. (2011) also found that some participants were able to integrate the treatment principles of ICBT for depression to improve daily life. In the present study an improvement in sleep and the ability 
Beukes et al. Participants' experiences of iCBT for tinnitus

to relax was noted. Participants also mentioned fewer participation restrictions and avoidance behaviours. This has also been reported by Thorén et al. (2014) for participants undertaking an online rehabilitative intervention for hearing aid users. In the context of a tinnitus intervention, this is an important outcome as avoidance behaviours are often associated with increased tinnitus distress (Beukes et al. 2017a; Hesser and Andersson 2009; Kleinstäuber et al. 2013). The strategies provided helped participants with general problem solving and the management of stress and anxiety. Similar effects of increased self-esteem due to becoming more knowledgeable about hearing loss were identified by those undertaking and IAR intervention (Malmberg et al. 2018). These effects are not always easy to measure using outcome measures, but are important to identify.

\section{Limitations}

The results of this study need to be interpreted with consideration of the sampling procedures used. Not all participants contacted chose to participate and only those willing to be interviewed participated. It is possible that those with stronger negative experiences were less inclined to participate. Furthermore, the sample was biased to those self-selecting to do an Internet-intervention. The overall more negative viewpoints of the intervention may thus not have been captured. Participant may also not have disclosed all negative experiences when being interviewed by someone unfamiliar.

\section{Clinical and research implications}

This study is the first to our knowledge identifying participants' views of ICBT for tinnitus. It indicated the potential of ICBT as a patient-centered care approach in reducing the impact of tinnitus though a comprehensive and accessible intervention. Despite the known efficacy of CBT in reducing tinnitus-related distress, clinical provision is sparse (McFerran et al. 2018). 
Beukes et al. Participants' experiences of iCBT for tinnitus

Improving access to CBT via the Internet is one way of improving patient centered care. The positive experiences of participants regarding the benefit of the intervention may also aid acceptability of such interventions from both professional and patients' viewpoints. Themes that were highlighted, such as the value of guidance, require further exploration to identify which aspects thereof aid engagement and positive outcomes. Further qualitative studies longitudinally following experiences while undertaking an Internet-based intervention should be investigated.

\section{Acknowledgements}

Our thanks is extended to the participants who gave up their time for these interviews.

Funding details not applicable

\section{Declaration of interest}

Anglia Ruskin, Lamar and Linköping Universities, and National Institute for Health Research, UK, supported the undertaking of this study but the views expressed are those of the authors and not of these institutions. No potential conflicts of interests are declared.

\section{Figure Captions}

Figure 1. Word cloud of the most frequently used words when describing the experiences with the intervention. A word that stands out more was more highly represented. 
Beukes et al. Participants' experiences of iCBT for tinnitus

Figure 2. Summary of the identified themes and sub-themes regarding participant experiences with iCBT for tinnitus

\section{Table}

Table 1: Participant characteristics

Table 2: Pre-intervention expectations and motivation

Table 3: Experiences of undertaking the intervention

Table 4: Factors affecting engagement

Table 5: Post-intervention effects

\section{Supplementary materials}

Appendix1: COREQ (COnsolidated criteria for REporting Qualitative research) Checklist

Appendix 2: Semi-structured interview guide

\section{References}

Andersson, G. 2002. Psychological Aspects of Tinnitus and the Application of Cognitivebehavioral Therapy. Clinical Psychology Review 22 (7): 977-990.

Andersson, G. 2015. Clinician-Supported Internet-Delivered Psychological Treatment of Tinnitus. American Journal of Audiology 24 (3): 299-301.

Andersson, G., T. Strömgren, L. Ström, and L. Lyttkens. 2002. Randomized Controlled Trial of Internet-Based Cognitive Behavior Therapy for Distress Associated with Tinnitus. Psychosomatic Medicine 64 (5): 810-816.

Andersson, G., and N. Titov. 2014. Advantages and Limitations of Internet-based Interventions for Common Mental Disorders. World Psychiatry 13 (1): 4-11.

Baguley, D., D. McFerran, and D. Hall. 2013. Tinnitus. The Lancet 382 (9904): 1600-1607.

Baumeister, H., L. Reichler, M. Munzinger, and J. Lin. 2014. The Impact of Guidance on Internet-Based Mental Health interventions: A Systematic Review. Internet Interventions 1 (4): 205-215. 
Beukes et al. Participants' experiences of iCBT for tinnitus

Beattie, A., A. Shaw, S. Kaur, and D. Kessler. 2009. Primary-care Patients' Expectations and Experiences of Online Cognitive Behavioural Therapy for Depression: A Qualitative Study. Health Expectations 12 (1): 45-59.

Bendelin, N, H. Hesser, J. Dahl, P. Carlbring, K.Z. Nelson, and G Andersson. 2011. Experiences of Guided Internet-Based Cognitive-Behavioural Treatment for Depression: A Qualitative Study. BMC Psychiatry 11 (1): 107.

Beukes, E.W., P.A. Allen, D.M. Baguey, V. Manchaiah, and G. Andersson. In press. LongTerm Efficacy of Audiologist-Guided Internet-Based Cognitive Behaviour Therapy for Tinnitus. The American Journal of Audiology.

Beukes, E.W., D.M. Baguley, P.M. Allen, V. Manchaiah, and G. Andersson. 2018. Audiologist-Guided Internet-Based Cognitive Behavior Therapy for Adults with Tinnitus in the United Kingdom: A Randomized Controlled Trial. Ear and Hearing 39:423-433.

Beukes, E.W., V. Manchaiah, P.M. Allen, D.M. Baguley, and G. Andersson. 2015. InternetBased Cognitive Behavioural Therapy for Adults with Tinnitus in the UK: Study Protocol for a Randomised Controlled Trial. BMJ Open 5 (9): e008241-2015-008241. doi:10.1136/bmjopen-2015-008241 [doi].

Beukes, E.W., V. Manchaiah, G., Andersson, P.M. Allen, P.M. Terlizzi, and D.M. Baguley. 2017a. Situationally Influenced Tinnitus Coping Strategies: A Mixed Methods Approach. Disability and Rehabilitation: 1-11.

Beukes, E.W., V. Manchaiah, D.M. Baguley, P.M. Allen, and G. Andersson. 2017b. Process Evaluation of Internet-Based Cognitive Behavioural Therapy for Adults with Tinnitus in the Context of a Randomised Control Trial. International Journal of Audiology: 1-12.

Beukes, E.W., V. Manchaiah, T.E. Valien, D.M. Baguley, P.M. Allen, and G. Andersson. 2017c. Positive Experiences Related to Living with Tinnitus: A Cross-sectional Survey. Clinical Otolaryngology 00:1-7.

Beukes, E.W., G. Vlaescu, V. Manchaiah, D.M. Baguley, P.M. Allen, V. Kaldo, and G. Andersson. 2016. Development and Technical Functionality of an Internet-Based Intervention for Tinnitus in the UK. Internet Interventions 6: 6-15.

Braun, V. and V. Clarke. 2006. Using Thematic Analysis in Psychology. Qualitative Research in Psychology 3 (2): 77-101.

Collins, Alf. 2014. Measuring what really Matters. Towards a Coherent Measurement System to Support Personcentred Care.London: The Health Foundation.

Craig, P., P. Dieppe, S. Macintyre, S. Michie, I. Nazareth, M. Petticrew, and Medical Research Council Guidance. 2008. Developing and Evaluating Complex Interventions: The New Medical Research Council Guidance. BMJ (Clinical Research Ed.) 337: a1655. doi:10.1136/bmj.a1655 [doi]. 
Beukes et al. Participants' experiences of iCBT for tinnitus

Eaton, S., S. Roberts, and B. Turner. 2015. Delivering Person Centred Care in Long Term Conditions. BMJ (Clinical Research Ed.) 350: h181. doi:10.1136/bmj.h181 [doi].

Essery, R, S. Kirby, A.W.A Geraghty, and L. Yardley. Older adults' experiences of internetbased vestibular rehabilitation for dizziness: A longitudinal study. Psychology \& health 32, no. 11 (2017): 1327-1347.

Fossey, E., C. Harvey, F. McDermott, and L. Davidson. 2002. Understanding and Evaluating Qualitative Research. Australian and New Zealand Journal of Psychiatry 36 (6): 717 732.

Hall, D.A., K. Fackrell, A.B. Li, R. Thavayogan, S. Smith, V. Kennedy, C. Tinoco et al. 2018. A narrative synthesis of research evidence for tinnitus-related complaints as reported by patients and their significant others. Health and quality of life outcomes 16 (1): 61 .

Heinrich, S., A. Rozental, P. Carlbring, G. Andersson., K. Cotter, and C. Weise. 2016. Treating tinnitus distress via the Internet: A mixed methods approach of what makes patients seek help and stay motivated during Internet-based cognitive behavior therapy. Internet Interventions 4: 120-130.

Hesser, H. and G. Andersson. 2009. The Role of Anxiety Sensitivity and Behavioral Avoidance in Tinnitus Disability. International Journal of Audiology 48 (5): 295-299.

Hibbard, J.H. and J. Greene. 2013. What the Evidence shows about Patient Activation: Better Health Outcomes and Care Experiences; Fewer Data on Costs. Health Affairs 32 (2): 207-214.

Holst, A., S. Nejati, C. Björkelund, M.C. Eriksson, M.C., D. Hange, M. Kivi, et al. 2017. Patients' experiences of a computerised self-help program for treating depression-a qualitative study of Internet mediated cognitive behavioural therapy in primary care. Scandinavian journal of primary health care 35 (1): 46-53.

Jasper, K., C. Weise, I. Conrad, G. Andersson, W. Hiller, and M. Kleinstaeuber. 2014. Internet- Based Guided Self-Help Versus Group Cognitive Behavioral Therapy for Chronic Tinnitus: A Randomized Controlled Trial. Psychotherapy and Psychosomatics 83 (4): 234-246. doi:10.1159/000360705.

Johansson, O., T. Michel, G. Andersson, and B. Paxling, 2015. Experiences of non-adherence to Internet-delivered cognitive behavior therapy: a qualitative study. Internet Interventions: 2(2): 137-142.

Kleinstäuber, M., K. Jasper, I. Schweda, W. Hiller, G. Andersson, and C. Weise. 2013. The Role of Fear-Avoidance Cognitions and Behaviors in Patients with Chronic Tinnitus. Cognitive Behaviour Therapy 42 (2): 84-99.

Ly, K.H., E. Janni, R. Wrede, M. Sedem, T. Donker, P. Carlbring, and G. Andersson. 2015. Experiences of a Guided Smartphone-Based Behavioral Activation Therapy for Depression: A Qualitative Study. Internet Interventions 2 (1): 60-68. 
Beukes et al. Participants' experiences of iCBT for tinnitus

Malmberg, M., E.S. Thorén, M. Öberg, T. Lunner, G. Andersson, and K. Kähäri. 2018. Experiences of an Internet-Based Aural Rehabilitation (IAR) Program for Hearing Aid Users: A Qualitative Study. International Journal of Audiology: 1-7.

Manchaiah, V., E.W. Beukes, S. Granberg, N. Durisala, D.M. Baguley, P.M. Allen, and G. Andersson. 2018. Problems and Life Effects Experienced by Tinnitus Research Study Volunteers: An Exploratory Study using the ICF Classification. Journal of the American Academy of Audiology. doi: 10.3766/jaaa.17094.

McFerran, D., D.J. Hoare, S. Carr, J. Ray, and D. Stockdale. 2018. Tinnitus services in the United Kingdom: a survey of patient experiences. BMC health services research, 18(1), p.110.

Meikle, M.B., J.A. Henry, S.E. Griest, B. J. Stewart, H.B. Abrams, R. McArdle, P. J. Myers, et al. 2012. The Tinnitus Functional Index: Development of a New Clinical Measure for Chronic, Intrusive Tinnitus. Ear and Hearing 33 (2): 153-176. doi:10.1097/AUD.0b013e31822f67c0.

Muller, I., S. Kirby, and L. Yardley. 2015. Understanding Patient Experiences of SelfManaging Chronic Dizziness: A Qualitative Study of Booklet-Based Vestibular Rehabilitation, with Or without Remote Support. BMJ Open 5 (5): e007680-2015007680. doi:10.1136/bmjopen-2015-007680.

O’Connor, S., P. Hanlon, C.A. O’Donnell, S. Garcia, J. Glanville, and F.S. Mair. 2016. Understanding Factors Affecting Patient and Public Engagement and Recruitment to Digital Health Interventions: A Systematic Review of Qualitative Studies. BMC Medical Informatics and Decision Making 16 (1): 120.

Oates, Julian, W. Wayne Weston, and John Jordan. 2000. The Impact of Patient-Centered Care on Outcomes. Fam Pract 49 (9): 796-804.

QSR International Pty Ltd. 2010. NVivo Qualitative Data Analysis Software. (Version 11.4).

Richards, T., A. Coulter, and P. Wicks. 2015.Time to Deliver Patient Centred Care. BMJ 2015;350:h530

Stacey, D., C.L. Bennett, M.J. Barry, N.F. Col, K.B. Eden, M. Holmes-Rovner, H. Llewellyn-Thomas, A. Lyddiatt, F. Légaré, and R. Thomson. 2011. Decision Aids for People Facing Health Treatment Or Screening Decisions. Cochrane Database Syst Rev 10 (10).

Teddlie, C., and F. Yu. 2007. Mixed Methods Sampling: A Typology with Examples. Journal of Mixed Methods Research 1 (1): 77-100.

Thorén, E.S., M. Öberg, G. Wänström, G. Andersson, and T. Lunner. 2014. A randomized controlled trial evaluating the effects of online rehabilitative intervention for adult hearing-aid users. International Journal of Audiology 53(7): 452-461. 
Beukes et al. Participants' experiences of iCBT for tinnitus

Tong, A., P. Sainsbury, and J. Craig. 2007. Consolidated Criteria for Reporting Qualitative Research (COREQ): A 32-Item Checklist for Interviews and Focus Groups.

International Journal for Quality in Health Care 19 (6): 349-357.

Yardley, L., B.J. Spring, H. Riper, L.G. Morrison, D.H. Crane, K. Curtis, G.C. Merchant, F. Naughton, and A. Blandford. 2016. Understanding and Promoting Effective Engagement with Digital Behavior Change Interventions. American Journal of Preventive Medicine 51 (5): 833-842. 